in terms of their immune cells profiles (i.e. their numbers, activation status etc.). Additionally, we were able to follow specific immune cell patterns inside the OA group. Our study further emphasized the role of immune cells in the pathogenesis of OA. In particular, the results provide evidence suggesting ongoing activation of innate immunity as well as a shift towards $T$ helper lymphocytes in fluids of the patients with OA. Our findings give a rational starting point to be addressed by the future research for clinically useful biomarkers associated with the OA development and progression.

Acknowledgements: Grant support: MZ CR VES16-31852A, MZ CR VES1527726A.

Disclosure of Interest: None declared

DOI: 10.1136/annrheumdis-2017-eular.4045

\section{SAT0515 SONOGRAPHIC AND CLINICAL EXAMINATION OF TENDON INVOLVEMENT IN HAND OSTEOARTHRITIS}

I. GessI, A. Vinatzer, G. Supp, A. Kerschbaumer, P. Studenic, M. Zauner, D. Aletaha, J. Smolen, P. Mandl. Dep. of Medicine 3, Div. of rheumatology, Medical University of Vienna, Vienna, Austria

Background: Persistent tenosynovitis and degenerative tendinitis is associated with pain and can lead to dysfunction and tendon damage. While tenosynovitis is a common finding in rheumatoid arthritis, data on tendon involvement in hand osteoarthritis (HOA) is clearly limited. The clinical assessment of tendons is difficult and not fully standardized, but muculoskeletal ultrasound (MSUS) has been used successfully in inflammatory rheumatic disease as a sensitive method to detect tenosynovitis, tendon damage and osteophytes.

Objectives: To characterize tendon involvement in hand osteoarthritis and compare ultrasound with clinical assessment.

Methods: In this cross-sectional observational study 34 patients with HOA underwent MSUS and clinical examination on the same day. Each flexor and extensor tendon of the hand was scored independently for tenosynovitis and tendon damage (presence/absence) respectively by an expert in MSUS, blinded to the results of the clinical examination. Additionally, osteophytes in the proximal and distal interphalangeal joints of the fingers were assessed. Clinical assessment of tendons for tendon involvement included volar or dorsal pain, crepitus and swelling involving the hand, wrist or forearm during active movement of the tendon against resistance according to the Birmingham consensus criteria, by assessors who were blinded to the results of the MSUS. Conventional radiographs (CR) of the hands were also acquired and evaluated by the Interphalangeal Osteoarthritis Radiographic Simplified score.

Results: The majority of patients $(30 / 34,88.2 \%)$ were female, with a mean age of $69.5 \pm 8.5$ years and a median of $10(9-22.5)$ years disease duration. Clinical examination revealed tendon involvement in $21(61.8 \%)$ patients with a median of 3 (range 1-14) affected tendons. In MSUS tenosynovitis was detected in $17 / 33$ patients $(51.5 \%)$ with a median of $1(1-4)$ tendon involved. Tendon damage was found in $8 / 33$ patients $(24.2 \%)$ with a median of $2(1-11)$ tendons involved. A total of $20 / 33(60.6 \%)$ patients exhibited sonographic signs of tendon involvement (tenosynovitis/tendon damage) with a median of $2(1-12)$ involved tendons. Tendon damage was found more often on the right hand $(p<0.05)$ while tenosynovitis did not significantly differ. Similarly, no significant difference between male and female patients was found. The extensor digitorum and the extensor carpi ulnaris tendons were the most commonly affected tendons under US. Tenosynovitis was found to be more prevalent among extensor tendons $(p<0.001)$, while tendon damage was demonstrated at a higher frequency in flexor tendons $(p<0.05)$ (Fig. 1). The agreement between MSUS and clinical examination was moderate on the patient level and poor on the level of individual tendons. Osteophytes were found in $96.8 \%$ patients using MSUS and in $100 \%$ of patients assessed using CR. Osteophytes detected on MSUS or CR showed good agreement $(p=0.01$, Cronbach's Alpha $=0.66)$.

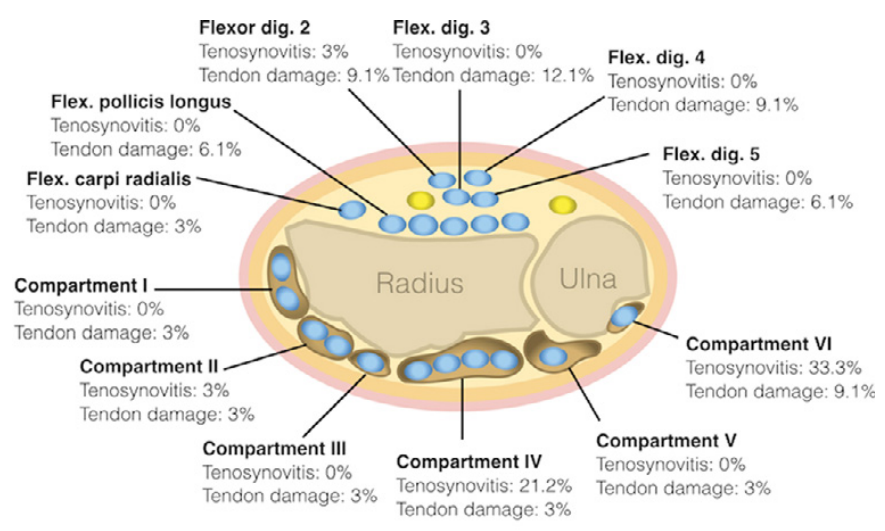

Fig.1 Distribution of tenosynovitis and tendon damage

Conclusions: The findings of our study reveal a high prevalence of tendon involvement in patients with hand OA. Sensitivity of MSUS in detecting tendon involvement coupled with the lack of agreement between clinical examination and MSUS on the level of individual tendons may suggest that while clinical examination is able to identify patients with overall tendon involvement, it does not allow the specific identification of involved tendons.

Disclosure of Interest: None declared

DOI: 10.1136/annrheumdis-2017-eular.5853

\section{SAT0516 INFLUENCE OF ETHNICITY ON CLINICAL PAIN IN PATIENTS WITH OSTEOARTHRITIS VERSUS RHEUMATOID ARTHRITIS: CONTRIBUTION OF LEVEL OF EDUCATION AND DEPRESSION}

I. Castrejon ${ }^{1}$, J. Chua ${ }^{1}$, J.A. Block ${ }^{1}$, L.A. Bradley ${ }^{2}$, Y. Yazici ${ }^{3}$, N. Shakoor ${ }^{1}$ ${ }^{1}$ Rheumatology, Rush University Medical Center, Chicago; ${ }^{2}$ Clinical Immunology and Rheumatology, University of Alabama, Birmingham; ${ }^{3}$ Rheumatology, NYU Hospital for Joint Diseases, New York, United States

Background: The experience of pain is characterized by inter-individual and group variability, with ethnicity being one potential contributing factor. In a systematic review, it was shown how African Americans (AA) demonstrated lower pain tolerance in experimental studies ${ }^{1}$. Pain is the most common and troubling symptom in patients with osteoarthritis (OA) -maybe reflecting damage- and in patients with rheumatoid arthritis (RA) -maybe reflecting synovitis- but other pain mechanisms are important.

Objectives: To investigate the influence of ethnicity on clinical pain and other outcomes and to identify potential predictors of higher scores for pain in AA and Hispanic patients with OA or RA seen in routine care.

Methods: As part of their clinic visit, all patients complete a multidimensional health assessment questionnaire (MDHAQ) at 2 academic sites. MDHAQ includes $0-10$ visual analogue scales (VAS) for pain, physical function, and a patient global evaluation (PATGL), and a depression score between others. The MDHAQ also include demographic data and patients "self-identify" their ethnicity. One random visit with complete questionnaire data for each $O A$ (ICD-9=715.0) and RA (ICD-9=714.0) patient from each site was included in this analysis. Comparison according to patients' self-reported ethnicity -White, African-American (AA) or Hispanics- were performed using ANOVA and chi-squared. Multiple regression models were performed to identify independent explanatory variables for clinical pain in AA and Hispanics groups versus White.

Results: The study included 402 OA patients and RA 373. There were no differences in age and gender between ethnicity groups in both diagnostic groups. Years of education were highest in the White followed by AA and then Hispanics in both $O A$ and RA. AA and Hispanics showed statistically significantly higher scores for pain (6.6 vs 5.3 in $O A, p<0.001 ; 5.7$ vs 4.4 in $R A, p<0.001)$ and lower physical function ( 3.2 vs 1.9 in $\mathrm{OA}, \mathrm{P}<0.001$; 3.2 vs 1.9 in $\mathrm{RA}, \mathrm{p}<0.001$ ) in comparison with Whites in both diagnostic groups. A lower level of education and a higher level of depression predicted greater pain on a MDHAQ in OA in separate models for AA and Hispanic patients and in RA in Hispanic patients (Table).

Table 1. Multiple regression models predicting clinical pain on a MDHAQ stratified by ethnicity

\begin{tabular}{|c|c|c|c|c|c|c|}
\hline \multirow[t]{2}{*}{ DV: MDHAQ-Pain (0-10) } & \multicolumn{3}{|c|}{ Osteoarthritis } & \multicolumn{3}{|c|}{ Rheumatoid Arthritis } \\
\hline & $\beta$ & $p$ & R squared & $\beta$ & $p$ & R squared \\
\hline \multicolumn{7}{|l|}{ Model 1} \\
\hline Age & -0.04 & 0.49 & $0.13^{*}$ & 0.08 & 0.25 & $0.10^{*}$ \\
\hline Sex & -0.07 & 0.21 & & 0.03 & 0.65 & \\
\hline African-Americans & 0.12 & 0.06 & & 0.04 & 0.94 & \\
\hline Education & -0.19 & 0.005 & & -0.09 & 0.19 & \\
\hline Depression & 0.19 & 0.002 & & 0.29 & $<0.001$ & \\
\hline \multicolumn{7}{|l|}{ Model 2} \\
\hline Age & -0.05 & 0.39 & $0.15^{\star}$ & 0.05 & 0.40 & $0.16^{\star}$ \\
\hline Sex & -0.12 & 0.07 & & 0.02 & 0.68 & \\
\hline Hispanic & 0.09 & 0.19 & & 0.03 & 0.63 & \\
\hline Education & -0.19 & 0.01 & & -0.19 & 0.01 & \\
\hline Depression & 0.21 & 0.002 & & 0.30 & $<0.001$ & \\
\hline
\end{tabular}

Conclusions: AA and Hispanic patients had higher level of pain than Whites, but these differences are mainly influenced by level of education and level of depression in OA and RA patients. These results support the biopsychosociocultural model of pain in which, ethnic group differences may be determined by multiple mechanisms including socio-cultural as education, and psychological as depression, in addition to biological pathways.

References:

[1] Rahim-Williams B, Riley JL, et al. Pain Med 2012, 13(4):522-540.

Disclosure of Interest: None declared

DOI: 10.1136/annrheumdis-2017-eular.3843 